\title{
Axion alert! Exotic-particle detector may miss out on dark matter
}

\section{Supercomputer calculation suggests hypothesized particle may be heavier than thought.}

Davide Castelvecchi

02 November 2016

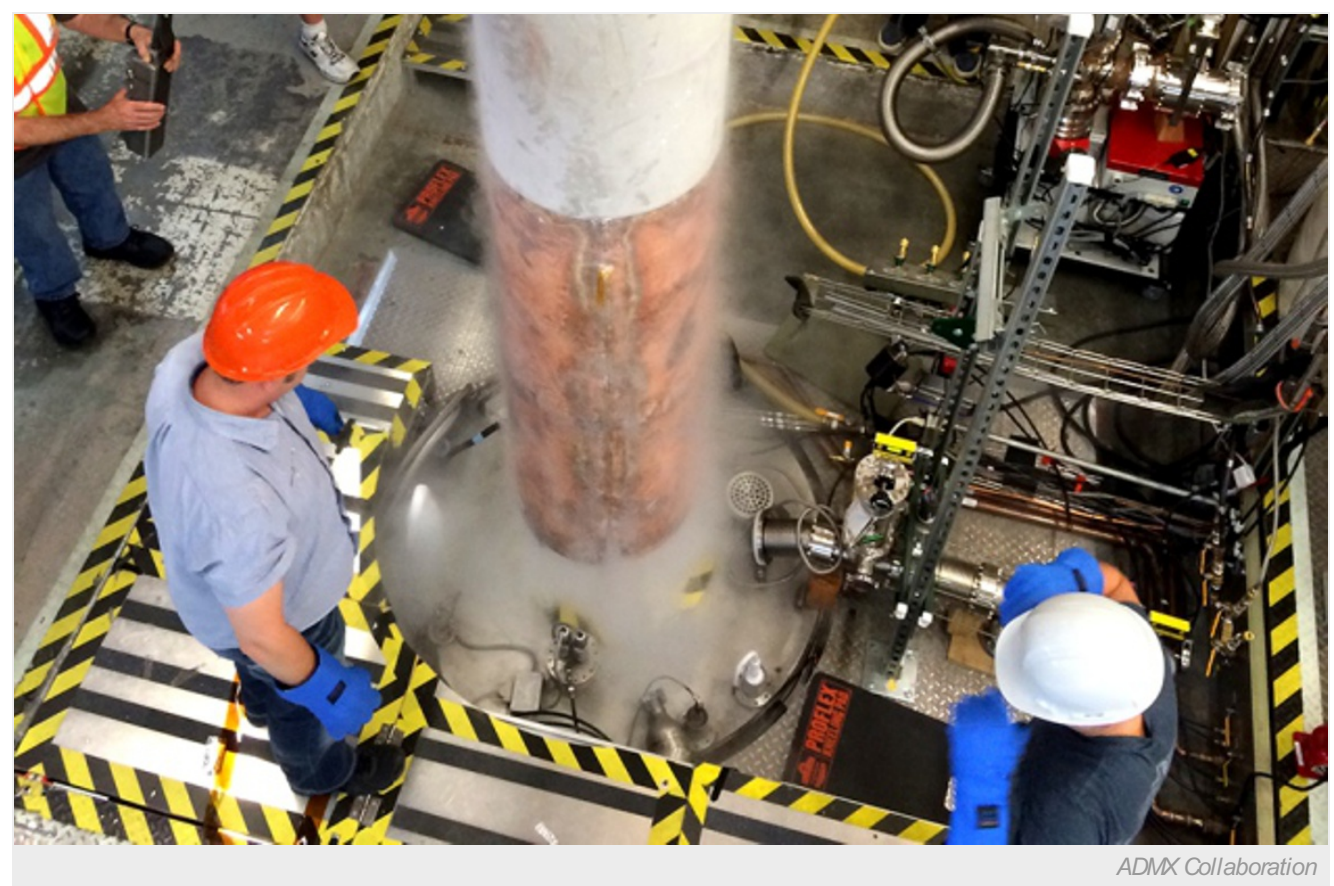

The AMDX experiment, a large magnet, is searching for hypothesized particles called axions.

An ambitious supercomputer calculation has brought good and bad news for physicists hunting the 'axion' - a hypothetical particle that is considered a leading candidate for dark matter.

The result shows that the axion, if it exists, could be at least ten times heavier than previously thought. If true, that's a useful clue on how to find the particle. But it also suggests that an experiment that has been hunting the axion for two decades might be unlikely to find it, because the detector was designed to search for a lighter version.

The axion was hypothesized in 1977 as a potential solution to a paradox arising from how the strong nuclear force — which binds particles in the nucleus of an atom together- affects antimatter and matter. (It would explain an unexpected symmetry whereby that force has the same effect on matter as it does on antimatter.) But researchers also think that the axion might be one of the components of dark matter, the invisible stuff thought to make up about $85 \%$ of the Universe's mass. So if the particle exists, it would solve two problems at once. 


\section{A hunt in the dark}

Physicists are desperately seeking the exotic particles that constitute dark matter, which has been spotted only indirectly, by its gravitational pull on galaxies. But so far, every experiment designed to find dark matter components has come up empty-handed or produced controversial results. Most of these experiments have tried to detect or produce so-called weakly interacting massive particles, or WIMPs.

The Axion Dark Matter eXperiment (ADMX) has been leading the charge for an alternative explanation: the axion. The experiment consists of a metal cylinder that uses strong magnetic fields, which theoretically should convert some axions into photons that could be detectedin the radio-wave spectrum. It began at Lawrence Livermore National Laboratory in California in 1996, and moved to the University of Washington in Seattle in 2010.

Early calculations had suggested that the axion should have a mass of around 5 microelectronvolts ( $\mu \mathrm{eV}$ ), about 100 billion times lighter than the electron, says Pierre Sikivie, a theoretical physicist at the University of Florida in Gainesville. So he and his collaborators designed ADMX to be most sensitive to masses in that range.

But in a paper published on 2 November in Nature ${ }^{1}$, Zoltan Fodor, a theoretical physicist at the University of Wuppertal in Germany, and his collaborators report the results of a large, complicated calculation showing that — under certain assumptions — the axion's mass is most likely to fall in the range between 50 and $1,500 \mu \mathrm{eV}$. That would put it outside the range of ADMX, which is currently sensitive to masses between about $0.5 \mu \mathrm{eV}$ and $40 \mu \mathrm{eV}$. "I think it is not very good news for ADMX," Fodor says.

\section{A matter of inflation}

The team used a supercomputer at the Jülich Supercomputing Centre in Germany to simulate the creation of elementary particles moments after the Big Bang, going back to times when temperatures soared above a million billion degrees, ten times hotter than those achieved in previous simulations. This was when axions - if they exist — would have been produced in abundance. To get there, the team had to develop techniques to speed up their calculations, which otherwise might have taken millions of years to complete, says Fodor.

The simulation allowed the team to calculate the axion's mass, under the assumption that it was created after inflation — a brief period during which the Universe expanded at an exponential rate. In a scenario in which the axion was created before inflation, the team worked out the way the particle would have formed, but were not able to deduce its mass.

The paper is "a tour de force", says Leslie Rosenberg, an experimental physicist at the University of Washington who leads ADMX. "My hats off to these people." If the post-inflation scenario is correct, then the team's results could mean that his experiment won't detect anything, he admits. But he adds that, in the pre-inflation case, what Fodor's team found could actually make a lighter mass more plausible, and would put it right in ADMXs "sweet spot".

Another detector being built at the Center for Axion and Precision Physics Research in Daejeon, South Korea, may be sensitive to higher masses, and so would the proposed MADMAX experiment, which was first suggested by one of the co-authors of the Nature paper.

Guido Martinelli, a theoretical physicist at the Sapienza University of Rome, agrees that it is too soon to write off ADMXs hunt entirely. Researchers are not sure which of the two scenarios — pre- or post-inflation — is more plausible, he says. Moreover, he argues, the team's results are not conclusive, even in the post-inflation scenario. "Some of their assumptions are a bit hasty," he says. "It is certainly necessary for other groups to verify these results."

Nature I doi:10.1038/nature.2016.20925

Read the related News \& Views article, "Particle physics: Axions exposed".

\section{References}

1. Borsanyi, S. et al. Nature http://dx.doi.org/10.1038/nature20115 (2016).

Nature ISSN0028-0836 ESSN 1476-4687

\section{SPRINGER NATURE}

